\title{
Функциональные возможности электромагнитно- акустических преобразований в токовом режиме в металлическом расплаве
}

\author{
В. Н. Цуркин, А. В. Иванов ${ }^{*}$ \\ Институт импульсных процессов и технологий НАН Украины, \\ 2. Николаев, 54018, Украина ${ }^{*}$ e-mail: artiomsan@gmail.com \\ Поступила в редакцию 24.11.2020 \\ После доработки 27.04.2021 \\ Принята к публикации 30.04.2021
}

\begin{abstract}
На основе физически обоснованных оценок выявлены ключевые характеристики процессов последовательного электромагнитно-акустического преобразования (ЭМАП) энергии при протекании электрического тока через расплав для симметричной задачи. При этом учитываются параметры источника тока, электрического поля, магнитного поля и магнитного давления в скин-слое, параметры акустических возмущений в расплаве. Показано, что при постановке задачи ЭМАП в технологических приложениях ключевыми параметрами являются геометрия емкости с объектом обработки и материал формы, а при решении задачи параметры скин-слоя и временная зависимость разрядного тока. Доля энергии при формировании магнитного давления в скин-слое составляет порядка от $10^{-4}$ до $10^{-2}$ от величины энергии, запасаемой в конденсаторной батарее генератора импульсного тока. Величина этой доли зависит от периода разрядного тока пропорционально $\sqrt{T}$. При распространении акустических возмущений в расплаве основная доля потерь энергии определяется разницей акустических жесткостей материалов расплава и формы. Характеристики акустических возмущений в расплаве существенно зависят от периода тока и геометрии емкости. Частотные спектры давления звуковых волн при выбранных для анализа параметрах могут перекрывать диапазон до сотен килогерц, что является весомым основанием для реализации резонансных эффектов и активного формирования диссипативных структур. Акцентировано внимание на том, что эффекты ЭМАП проявляются в расплаве под воздействием не только акустического поля, но и электромагнитного в скин-слое. Они разделены по времени, но акустическое поле может занимать весь объем расплава, и его воздействие более продолжительно во времени.
\end{abstract}

Ключевые слова: электрический разряд, расплав, преобразование энергии, потери энергии, акустические волновые процессы, функциональные возможности

УДК 537.528:621.3.017:534.21

https://doi.org/10.52577/eom.2021.57.6.60

ВВЕДЕНИЕ

В технологиях литейного производства используют различные технологические способы обработки расплава в температурных интервалах от жидкого состояния до начала полного твердения. Эти способы можно поделить на реагентные и энергетические. В первом случае в расплав вводят те или иные реагенты, которые действуют избирательно. Например, Ті способствует измельчению зеренной структуры сплавов $\mathrm{Al}$, соли $\mathrm{Na}$ и (или) K способствуют модифицированию эвтектики, Mn активен по отношению к фазам Fe и т.п. Энергетические методы используют обработку полями различной физической природы. Их действие - многопараметрическое, многофункциональное и многоцелевое, поэтому энергетическое воздействие является кооперативным. Физическая природа энергетического воздействия не ограничивается только влиянием энергии базового поля. В объекте обработки, как правило, формируются вторичные поля.
Физической основой таких преобразований является хорошо изученные механизмы электромеханических, электромагнитных, термоэлектрических, термоакустических и т.п. трансформаций. $\mathrm{B}$ их числе электромагнитноакустические преобразования (ЭМАП), которые происходят в условиях скинирования электромагнитной энергии, когда глубина скин-слоя $\delta$ меньше размеров объекта обработки.

Для технологических приложений решаемые задачи ЭМАП сводятся к изучению условий и факторов возбуждения акустических волн давлением магнитного поля, которое генерируется в скин-слое при протекании импульсов электрического тока. Далее решаются задачи распространения акустических возмущений и их взаимодействия с преградами (стенки технологического реактора) и фазами объекта обработки (неоднородностями). В итоге прогнозируется конечный эффект акустического воздействия.

Решение такой задачи методами моделирования даже в упрощенных постановках 
сталкивается, прежде всего, с нехваткой экспериментальных данных, тем более что такие задачи квалифицируются как мультифизические, так как нужно анализировать многофакторные, взаимообусловленные кооперативные процессы разной физической природы, механизмы и природа которых являются зачастую дискуссионными.

Поэтому для металлического расплава изучение соответствующей проблемы находится в инкубационном состоянии. Ее анализ ограничивается (судя по публикациям) решением отдельных задач [1-7], основная цель которых обозначить некоторые явления на отдельных этапах ЭМАП. При этом расплав нагружается или индуктором [1-4], или пропусканием тока непосредственно через расплав [5, 6]. Формируемые при этом эффекты в основном поясняются только косвенно действием акустических возмущений. Или же априори анализируются функциональные возможности ЭМАП без привязки к способу формирования магнитного давления в скин-слое [7-11], показывая качественную формализованную картину физических механизмов ЭМАП. Но, как подчеркнуто в работе [11], отдельные детали этой картины найдены пока лишь теоретически и ждут своего экспериментального подтверждения.

В этом плане актуальной представляется задача, в которой на уровне оценок учитываются основные ее положения при последовательных преобразованиях энергии при ЭМАП. Такой подход позволит проанализировать функциональные возможности ЭМАП при использовании способа обработки расплава импульсами электрического тока.

В данной работе на основе физически обоснованных оценок процессов ЭМАП энергии, генерируемых при пропускании электрического импульсного тока через металлический расплав, связаны ключевые параметры в системе: «параметры источника тока-параметры электромагнитного поля и магнитного давления в скинслое-параметры акустических возмущений»».

Такой подход прежде всего преследовал цель - дополнить известные результаты в отношении ЭМАП для развития научных основ этого интересного явления применительно к обработке расплава импульсами тока в технологиях литейного производства.

\section{СОСТОЯНИЕ РАЗРАБОТКИ ПРОБЛЕМЫ}

При использовании способа энергетической обработки расплава следует учитывать, что внешнее энергетическое воздействие по своему назначению можно классифицировать как формирующее, инициирующее и активирующее.
В первом случае плотность энергии воздействия должна быть сопоставима с плотностью внутренней энергии системы как объекта обработки. Во втором случае энергия воздействия инициирует те процессы, которые не характерны для состояния объекта обработки (аналогично действию прекурсоров). В третьем - процессы в объекте обработки активируются подобно действию катализаторов. Для второго и третьего случаев плотность энергии воздействия на порядки меньше, чем для первого. Основное воздействие здесь проявляется в основном через резонансные и (или) термофлуктуационные механизмы.

В ранних работах $[10,11]$ особо подчеркнуто, что энергия акустического поля составляет величину порядка $10^{-2}$ от энергии электрического поля. В работе [5] этот показатель оценен величиной порядка $10^{-8}$. То есть энергетические потери при ЭМАП оценены соответственно $10^{2}$ и $10^{8}$. Но следует отметить, что оба эти показателя в указанных работах представлены без физического обоснования энергетических аспектов всех этапов ЭМАП.

Безусловно, влияние акустических волн на объект обработки нужно рассматривать только на фоне воздействия электромагнитной энергии на проводящую среду - расплав. В этом случае следует обратиться к термину «совокупный экстремум воздействия» [7]. До сих пор его определяют пока postfactum в эксперименте.

К сожалению, в известных нам публикациях мы не нашли числовых данных, характеризующих в решаемых задачах объем (массу) обрабатываемого сплава и его геометрическую форму. Их совокупность является ключевым фактором в формировании скин-слоя, в котором генерируется «магнитный поршень», возбуждающий акустические волны. В свою очередь геометрия объекта обработки и его размеры влияют на процессы распространения волн. Кроме того, при постановке опубликованных задач не акцентируется внимание на электродной системе, которая служит для передачи энергии от источника тока в расплав. Ранее в работе [12] была показана существенная разница (качественная и количественная) в распределении электрического и магнитного полей при разных типах электродной системы.

Для литейного производства любой способ энергетического воздействия на расплав не должен существенно повышать цену конечной продукции, которая складывается из энергозатрат на обработку и стоимости энергетического комплекса. 


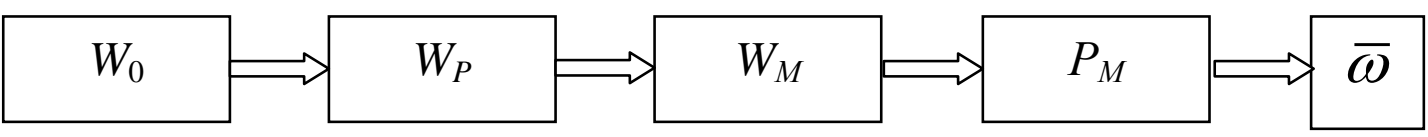

Рис. 1. Стадии формирования акустических волн.

Таким образом, решение научно-технической проблемы генерирования эффектов ЭМАП при электротоковой обработке расплава в литейном производстве имеет следующие особенности.

1. Мультифизический характер проблемы, обусловленный многопараметрическим, многофункциональным и многоцелевым воздействиями.

2. Роль акустического поля при ЭМАП в совокупности с электромагнитным воздействием в изменении кристаллизационной способности расплава тока однозначно не определена.

3. Влияние именно ЭМАП на соотношение «цена-качество» литой металлопродукции требует более активной дискуссии.

Но на сегодня эти особенности не умаляют решения частных задач, комплекс которых в дальнейшем позволит сформулировать научные основы указанной проблемы.

\section{ОЦЕНКА ПАРАМЕТРОВ ЭМАП В СИСТЕМЕ «ПАРАМЕТРЫ ИСТОЧНИКА \\ ТОКА-ПАРАМЕТРЫ ЛЕКТРОМАГНИТНОГО \\ ПОЛЯ И МАГНИТНОГО ДАВЛЕНИЯ \\ В СКИН-СЛОЕ-ПАРАМЕТРЫ \\ АКУСТИЧЕСКИХ ВОЗМУЩЕНИЙ»}

Формирование акустических волн в металлическом расплаве обеспечивается следующими стадиями (рис. 1).

Электрическая энергия, запасенная в конденсаторной батарее генератора импульсных токов, $W_{0}$ при электрическом разряде на нагрузку (расплав) трансформируется в энергию разряда $W_{P}$. При прохождении переменного электрического тока вихревое электрическое поле самоиндукции вытесняет его к поверхности в скин-слой. Если среда нагрузки не ферримагнитна (что логично, так как металлические расплавы имеют температуру, превышающую точку Кюри), то собственная энергия тока есть энергия его магнитного поля $W_{M}$, объемная плотность которой соответствует величине магнитного давления $P_{M}$. Его изменение во времени, коррелирующее с аналогичным процессом разрядного тока, формирует в расплаве акустические возмущения с удельной мощностью звука $\bar{\omega}$.

В случае геометрической постановки задачи в виде прямого цилиндра с электродами типа «стержень-стержень», которые расположены вдоль оси цилиндра, «магнитный поршень» сосредоточен равномерно в скин-слое, опоясывающем поверхность расплава.

В этой последовательности стадий на каждом этапе происходят потери энергии. Даже оценочный учет этих потерь позволит выявить потенциальные возможности разрядного тока формировать акустическое поле. Это даст возможность оценить требуемые величины параметров генератора импульсных токов.

Электрическая энергия, запасаемая в конденсаторной батарее генератора импульсных токов:

$$
W_{0}=\frac{C \times U_{0}^{2}}{2},
$$

где $U-$ напряжение на обкладках конденсатора, В; $C$ - емкость конденсатора, $\Phi$.

При разряде на нагрузку за счет потерь в RLC-контуре на его полном сопротивлении величина $W_{P}$, по данным экспериментов, может уменьшиться до значения:

$$
W_{P} \approx(0,2 \div 0,3) \times W_{0} .
$$

Собственная энергия тока есть энергия его магнитного поля:

$$
W_{M}=\frac{V_{c}}{2} \times \mu \times \mu_{0} \times \frac{I^{2}(t)}{(2 \times \pi \times r)^{2}},
$$

где $\mu, \mu_{0}-$ соответственно магнитная проницаемость и магнитная постоянная, Гн/м; I - амплитуда силы разрядного тока, А; $t$ - текущее время, с; $r$ - текущая координата, м; $V_{\text {с }}$ - объем скин-слоя, м ${ }^{3}$.

Энергия $W_{M}$ сосредоточивается в скин-слое. Его толщина:

$$
\delta=\frac{2}{\sqrt{2 \times \mu \times \mu_{0} \times \gamma \times \omega}},
$$

где $\gamma$ - проводимость материала расплава, $\left(\mathrm{Oм}^{\cdot}\right)^{-1} ; \omega-$ круговая частота, Гц $(\omega=2 \times \pi / T$, где $T$ - период тока, с).

В скин-слое величина тока падает от поверхности проводника в $e$ раз к его оси. В этом случае, если $\delta<<r$, можно оценить некое эффективное значение $\bar{W}_{M}$ в виде:

$$
\bar{W}_{M} \approx 3,8 \times 10^{-3} \times V_{c} \times \mu \times \mu_{0} \times I^{2}(t) / r^{2} .
$$

Для цилиндрической емкости с расплавом радиуса $r_{\kappa}$ высотой $2 r_{\kappa}$

$$
V_{c} \approx 6 \times \pi \times r^{2} \times \delta=26,6 \times r^{2} / \sqrt{\mu \times \mu_{0} \times \gamma \times \omega} .
$$


Тогда формула (5) примет вид:

$$
\bar{W}_{M} \approx 0,1 \times I^{2}(t) \times \sqrt{\frac{\mu \times \mu_{0}}{\gamma \times \omega}} .
$$

Здесь величина под знаком радикала имела размерность Гн. Ее физический смысл - индуктивность нагрузки, то есть расплава:

$$
L_{P} \approx \sqrt{\frac{\mu \times \mu_{0}}{\gamma \times \omega}} .
$$

Величина $\bar{W}_{M}$ определяется только величиной силы тока, магнитными характеристиками материала расплава, его проводимостью и циклической частотой тока. Изменение величины $\bar{W}_{M}$ наиболее чувствительно к изменению величины силы тока, которая, если переписать формулу (1) в иных терминах, может быть оценена соотношением:

$$
I_{0}^{2}=2 \times W_{0} / L_{K},
$$

где $L_{K}-$ индуктивность колебательного разрядного контура, Гн.

Тогда максимальное значение эффективной магнитной энергии:

$$
\bar{W}_{M} \approx 0,2 \times W_{0} \times L_{P} / L_{K} .
$$

Для металлических расплавов в соответствии с формулой (7) величина $L_{P}$ может быть оценена как $2 \times 10^{-7} \sqrt{T}$, где $T-$ период тока. Индуктивность разрядного контура генератора импульсных токов промышленного исполнения $L_{K}$ составляет величину порядка единиц мкГн. Тогда соотношение $L_{P} / L_{K} \approx 0,1 \times \sqrt{T}$.

В этом случае доля энергии $W_{P}$, формирующая в процессе разряда магнитное давление $P_{M}$, в соответствии с формулой (2) может составлять величину:

$$
\begin{aligned}
& d \approx \bar{W}_{M} / W_{P} \approx 0,2 \times W_{0} \times 10^{-1} \times \\
& \times \sqrt{T} /\left(0,2 \times W_{0}\right)=10^{-1} \times \sqrt{T} .
\end{aligned}
$$

Таким образом, ключевым параметром, определяющим в процессе разряда величину магнитного давления, является период разрядного тока.

Для токов микросекундного диапазона величина $d$ может соответствовать пределу от $10^{-4}$ до $10^{-3}$. Для периодов тока миллисекундного диапазона - от $10^{-3}$ до $10^{-2}$. То есть энергетические потери в цепочке преобразований от $W_{0}$ до $\bar{W}_{M}$ (рис. 1) в зависимости от периода тока могут лежать в пределах от $10^{4}$ до $10^{2}$.

Проанализируем зависимость магнитного давления $\bar{P}_{M}$ от параметров разряда и характе- ристик объекта обработки. С учетом формулы (5):

$$
\bar{P}_{M}(t) \approx 3,8 \times 10^{-3} \times \mu \times \mu_{0} \times I^{2}(t) / r^{2} .
$$

Его максимальное значение $\bar{P}_{M}^{\max }$ можно записать в виде:

$$
\bar{P}_{M}^{\max }(t) \approx 7,6 \times 10^{-3} \times \mu \times \mu_{0} \times W_{0} /\left(r^{2} \times L_{K}\right) .
$$

Сделаем некоторые оценки. Например, при $W_{0}=1$ кДж, $L_{K}=2 \times 10^{-6} \Gamma \mathrm{H}$, в емкости радиуса $r=0,1 \mathrm{M}$, величина $\bar{P}_{M}^{\max }=480$ Па. Тогда удельная мощность звука (энергия, передаваемая волной через единицу поверхности в единицу времени, - интенсивность звука):

$$
\begin{gathered}
\bar{\omega} \approx \frac{1}{2 \times \rho \times c} \times\left(\bar{P}_{M}^{\max }\right)^{2}=2 \times 10^{-2} \mathrm{BT} / \mathrm{M}^{2}, \text { или } \\
\bar{\omega} \approx 300 \mathrm{db} .
\end{gathered}
$$

Здесь $\rho \times c$ - акустическая жесткость материала расплава; $\rho$ - его плотность, кг $/ \mathrm{M}^{3} ; c$ - скорость звука, м/с.

Как видим из формулы (12), ключевым параметром в формировании максимального значения давления «магнитного поршня» является радиус емкости с расплавом. Наш пример скорее соответствует размеру поперечного сечения расплава в желобе при заливке формы для фасонной отливки или же ручья расплава при непрерывной разливке. В случае ЭМАП в печи или разливочном ковше увеличение $r$ активно уменьшит величину $\bar{P}_{M}^{\max }$, а значит, и эффект формирования амплитуды акустической волны.

Обратим внимание на формулу (11), содержащую зависимость $I(t)$. Длительность импульса тока $I(t)$ величиной $\tau_{\text {и }}$ определяет протяженность волнового пакета (цуга) $\lambda=\tau_{\mathrm{r}} c$, где $c$ - скорость звука. Учитывая то обстоятельство, что разряд на жидкий металл почти соответствует режиму короткого замыкания в разрядном контуре, ожидаем относительно длительный колебательный разряд. При величине $c \approx 5 \times 10^{3} \mathrm{~m} / \mathrm{c} \lambda$ может достигать величин, соизмеримых с размерами емкости промышленных условий. Учитывая то, что в металлическом расплаве затухание звуковых волн незначительно [13], можно ожидать существенного увеличения амплитуды давления при суперпозиции встречных волновых пакетов в центре емкости. Также может возникнуть ситуация полного заполнения емкости волновым полем.

При обработке расплава используется принцип нагружения повторяющимися импульсами с частотой $f, \mathrm{c}^{-1}$. В свою очередь, $W_{0} \times f=N_{\text {п }}$, где $N_{\text {п }}-$ мощность пакета 
импульсов, Вт. Потребляемая генератором импульсных токов мощность $N \approx N_{\text {п }} / k$, где $k \approx 0,3-0,6$, и определяет потери энергии в системе генератора импульсных токов [14]. В случае высоковольтных импульсов предпочтительно, чтобы значение $f<10 \mathrm{c}^{-1}$, что обусловлено особенностями реализации высоковольтных генераторов импульсных токов. Для низковольтных импульсов $f$ может ограничиваться величиной в несколько тысяч $\mathrm{c}^{-1}$ (при малых значениях $W_{0}$ ). В сопоставлении значений $W_{0}$ и $N_{\text {п }}$ с величиной $\bar{P}_{M}^{\max }$ в формуле (12) можно оценить соотношение потребляемой генератором энергии и реальных затрат энергии на обработку и эффект ЭМАП.

Необходимо отметить, что при анализе функциональных возможностей ЭМАП следует учитывать отражение и преломление волн как на внутренних и внешних стенках емкости, так и на свободной поверхности расплава. В этом случае, как известно, нужно сопоставить величины акустических жесткостей расплава $\rho_{1} c_{1}$ и преград $\rho_{2} c_{2}$. Такая задача уже не входит в рамки оценок, поэтому в данной работе она не рассматривалась.

Во многих цитируемых в статье источниках отмечается, что подбором частоты тока можно найти приемлемый показатель эффективности ЭМАП, к сожалению, это заявление декларативно. Наши оценки показали, что важнейшим параметром тока разряда для процессов ЭМАП является его циклическая частота $\omega$ (период $T$ ), которая, прежде всего, определяет геометрические размеры скин-слоя $\delta$ (4), а значит, и плотность энергии $\bar{W}_{M}$. Таким образом, временная зависимость $P_{M} \sim f\left(I^{2}(t)\right)$ содержит в формализованном виде размер величины $\omega$, что позволяет численным моделированием проанализировать роль циклической частоты (периода $T$ ) импульса разрядного тока в формировании акустического поля в расплаве $\mathrm{Al}$.

\section{ЧИСЛЕННЫЙ АНАЛИЗ РАСПРОСТРАНЕНИЯ АКУСТИЧЕСКОГО ВОЗМУЩЕНИЯ}

Ограничиваясь рамками данной статьи, рассмотрим в упрощенном виде следующую задачу.

В цилиндрической емкости с абсолютно жесткими стенками с помощью электродов, расположенных на оси цилиндра (симметричная задача), в расплаве $\mathrm{Al}$ произведен электрический разряд, величина тока которого формализована временной зависимостью:

$$
I(t)=I_{0} \times \sin (\omega \times t) \times \exp \left(-R \times t /\left(2 \times L_{K}\right)\right),
$$

здесь $\omega-$ циклическая частота, Гц; $R$ - омическое сопротивление контура, Ом; $L_{K}-$ индуктивность контура, $\Gamma$ н.
На рис. 2 представлена геометрическая постановка задачи. Здесь обозначения $A, B$ показывают анализируемые точки в расплаве. Принято: скорость звука в расплаве $c=4700 \mathrm{~m} / \mathrm{c}$, плотность расплава $\rho=2400 \kappa г / \mathrm{m}^{3}$.

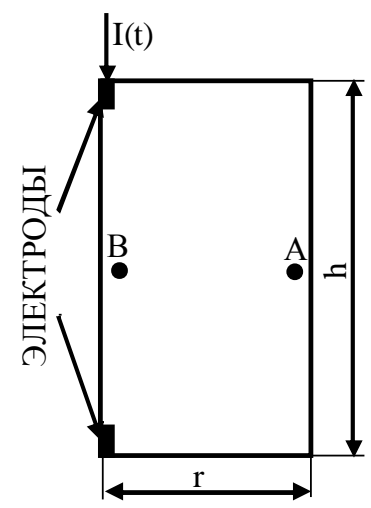

Рис. 2. Геометрическая постановка задачи.

Сравним последействие ЭМАП для двух импульсов тока с $\omega=2,2$ МГц $(T=2,8$ мкс) и $\omega=22$ МГц ( $T=28$ мкс). Также примем одинаковую длину цуга импульса тока $\tau_{\text {и }}=100$ мкс и одинаковый декремент затухания импульса тока в разрядном контуре $R / 2 L_{K}=25 \times 10^{3} \mathrm{c}^{-1}$.

Пусть в скин-слое цилиндрической емкости для обоих импульсов тока реализуются одинаковые импульсы магнитного давления $P_{M}(t)$, что достигается подбором величины $I_{0}$ в формуле (13). Пусть $P_{M}^{\max }=5$ кПа.

В этом случае решение акустической задачи в приближении сжимаемой невязкой жидкости не представляет трудностей.

Проанализируем для сравнения акустическую обстановку в двух емкостях, когда $r_{1}=4 \times 10^{-2} \mathrm{M}$; $h_{1}=9 \times 10^{-2}$ м и $r_{2}=0,4 \mathrm{м} ; h_{2}=0,9$ м. Соответственно массы расплава равны $\approx 1$ кг и $\approx 100$ кг. Первый вариант соответствует лабораторным экспериментам, другой - возможному промышленному случаю обработки.

Протяженность первичного волнового пакета $\lambda$ определяется длительностью импульса давления $\tau_{\text {и }}$ и скоростью звука в расплаве $c$ так, что $\lambda=\tau_{\mathrm{u}} \times c=0,47 \mathrm{м}$. То есть протяженность первичного волнового пакета практически в 10 раз больше величины радиуса емкости на 1 кг расплава и практически равна величине радиуса емкости на 10 кг расплава. Временной интервал перехода возмущения из точки $A$ в точку $B$ (рис. 2) для 1 кг составит 8,5 мкс. Для 100 кг 85 мкс. Эти параметры показывают возможную качественную и количественную разницу в суперпозиции волн, распространяющихся в разных объемах расплава.

Важно также отметить, что распространение происходит в цилиндрической емкости со сжимаемой жидкостью. Поэтому по мере распространения импульса давления плотность 


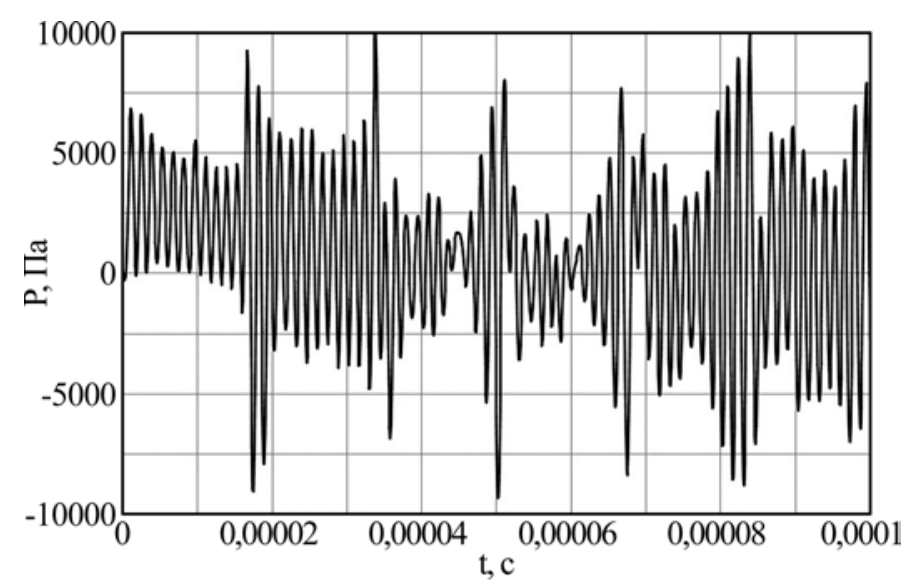

(a)

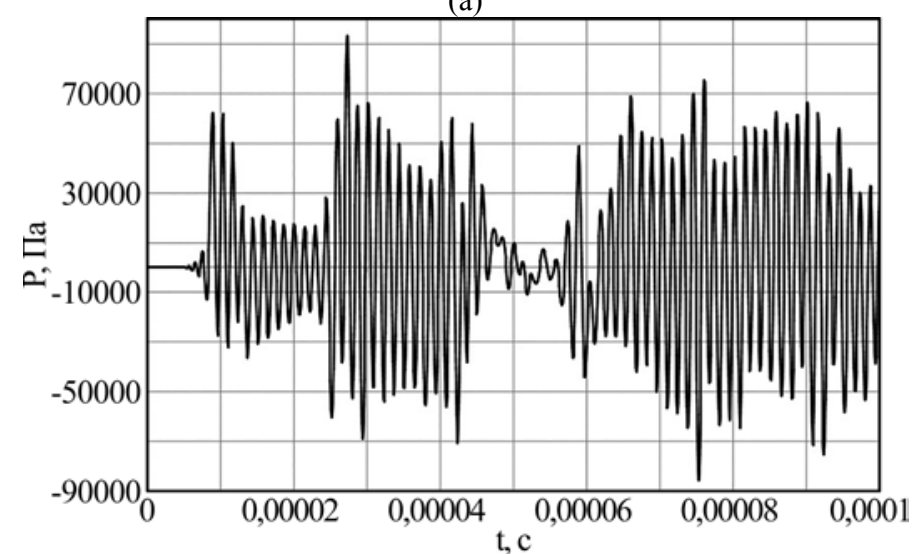

(б)

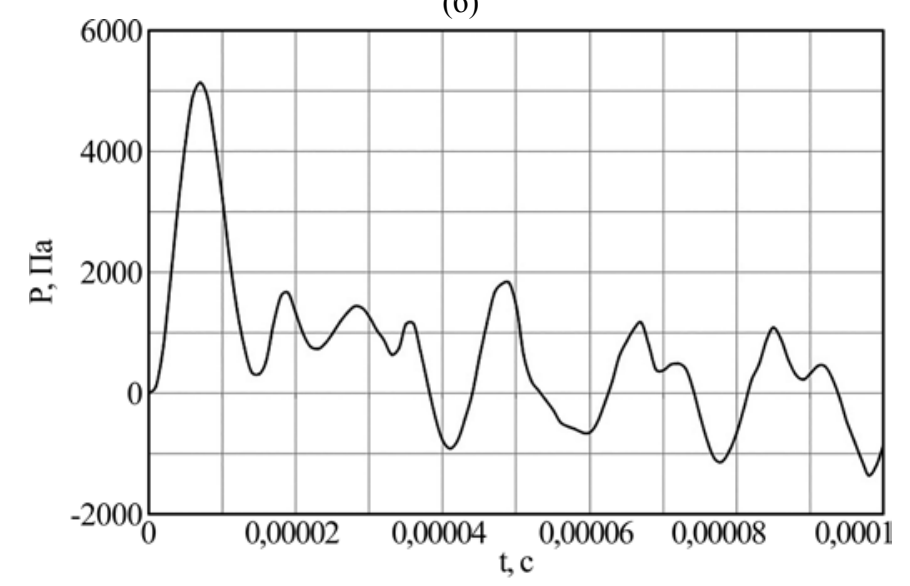

(в)

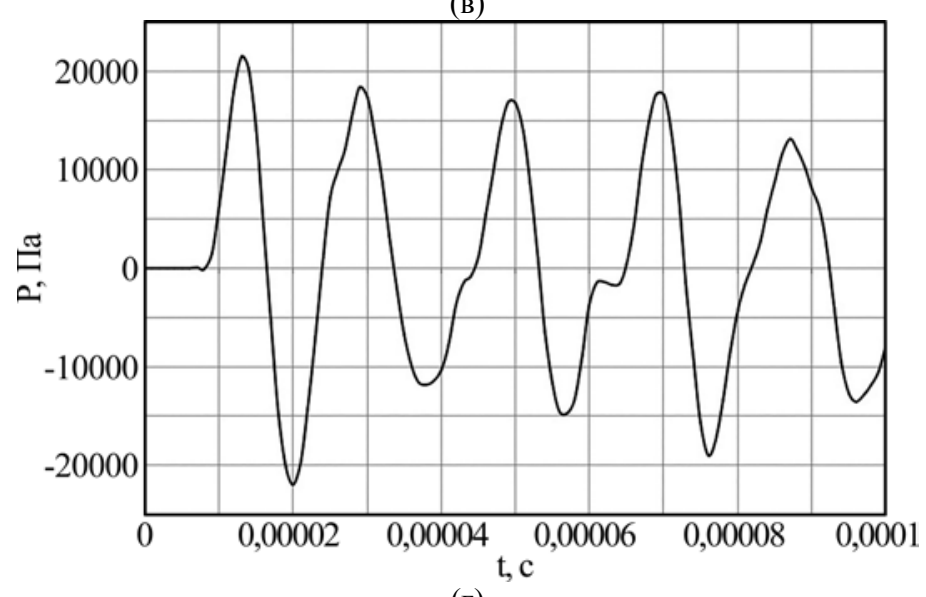

(г)

Рис. 3. Зависимости первичного сигнального давления в точке $A$ и давление в точке $B$, размеры емкости с расплавом $h=0,09$ м, $r=0,04$ м. (а) - первичное сигнальное давление в точке $A$ (период тока 2,8 мкс); (б) - давление в точке $B$ (период тока 2,8 мкс); (в) - первичное сигнальное давление в точке $A$ (период тока 28 мкс); (г) - давление в точке $B$ (период тока 28 мкс). 


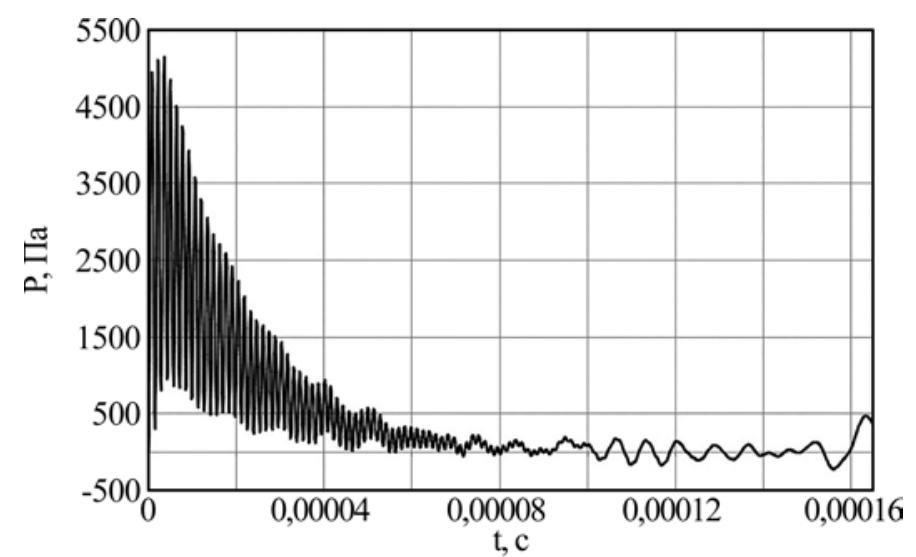

(a)

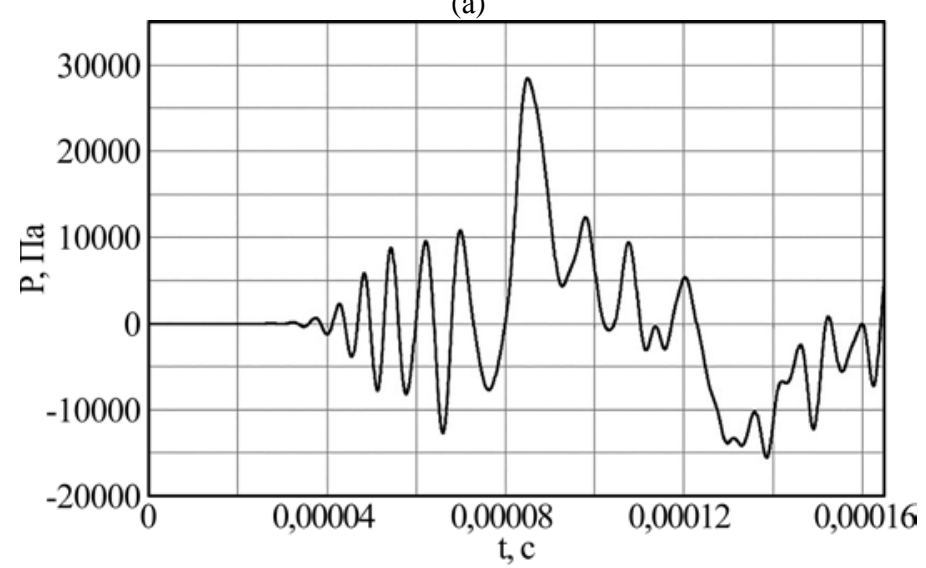

(б)

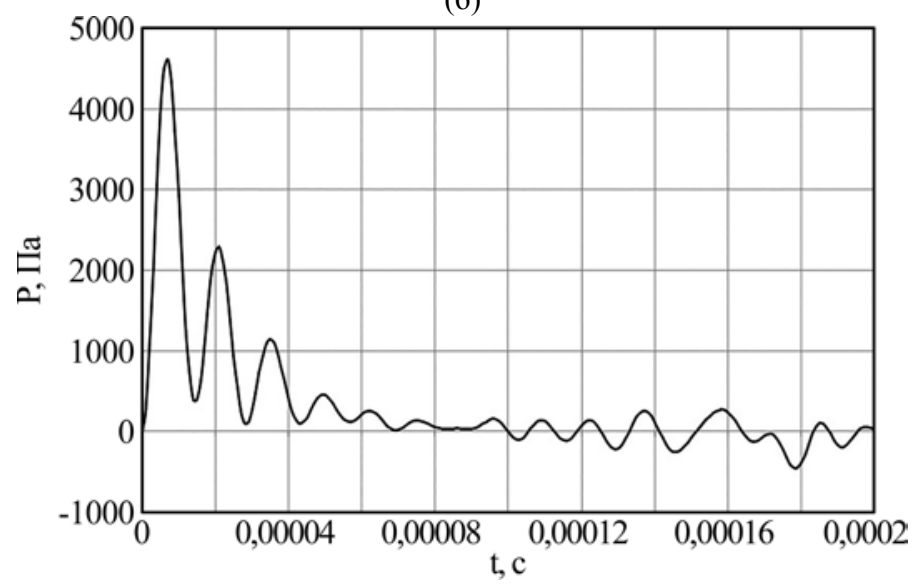

(B)

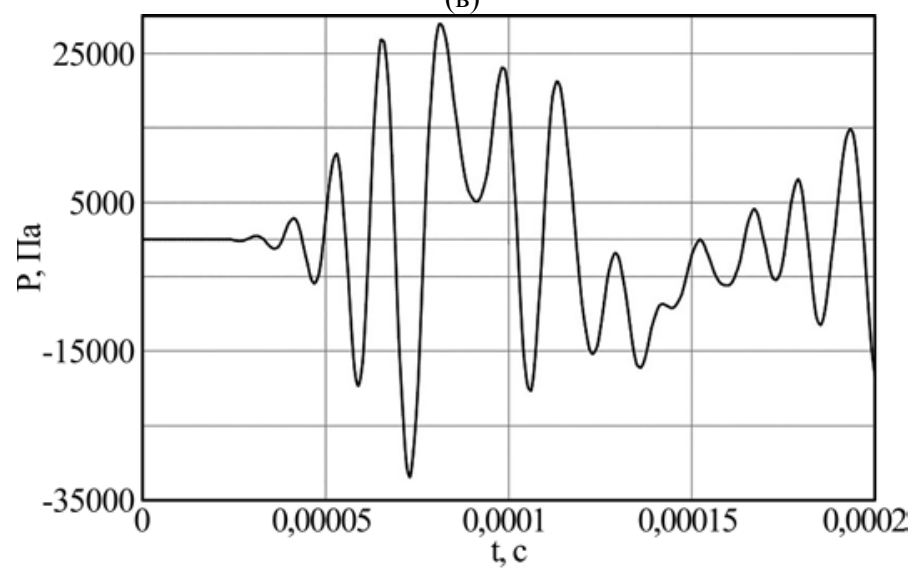

(г)

Рис. 4. Зависимости первичного сигнального давления в точке $A$ и давление в точке $B$, размеры емкости с расплавом $h=0,9$ м, $r=0,4$ м. (а) - первичное сигнальное давление в точке $A$ (период тока 2,8 мкс); (б) - давление в точке $B$ (период тока 2,8 мкс); (в) - первичное сигнальное давление в точке $A$ (период тока 28 мкс); (г) - давление в точке $B$ (период тока 28 мкс). 


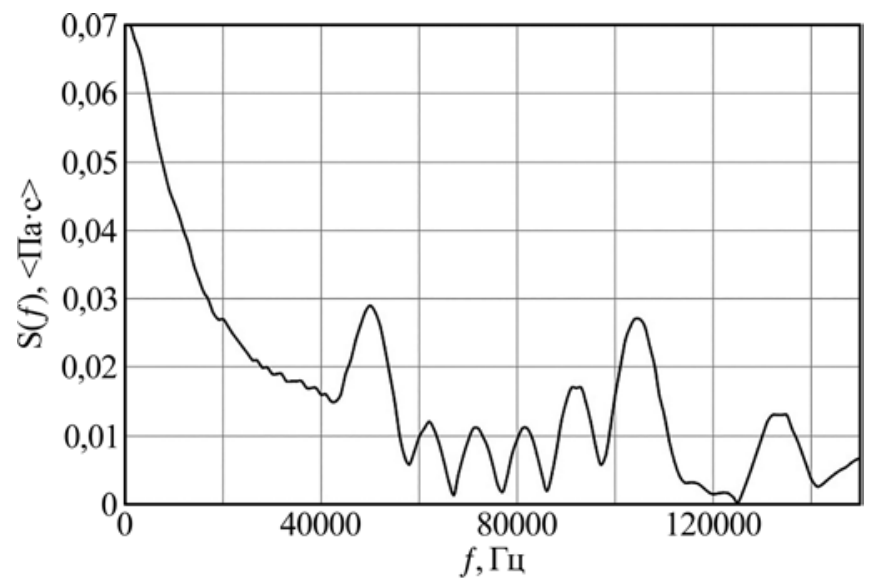

(a)

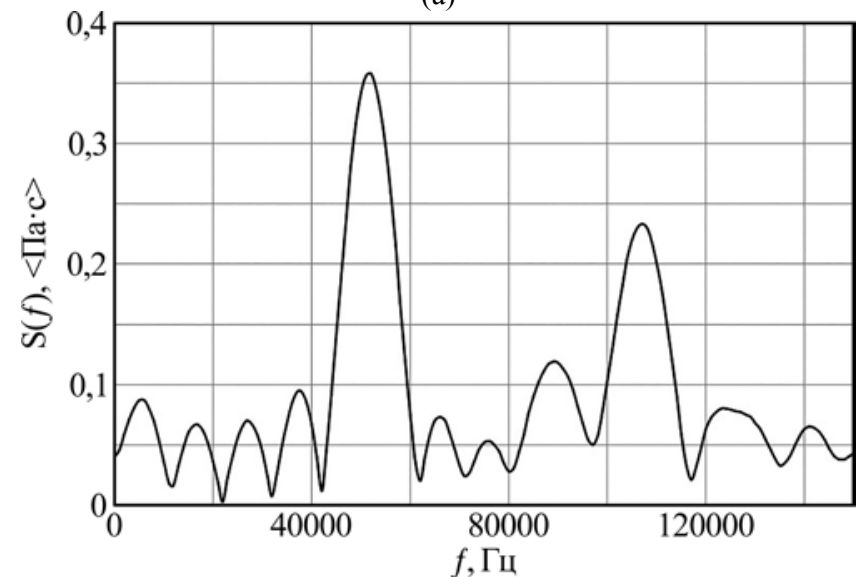

(б)

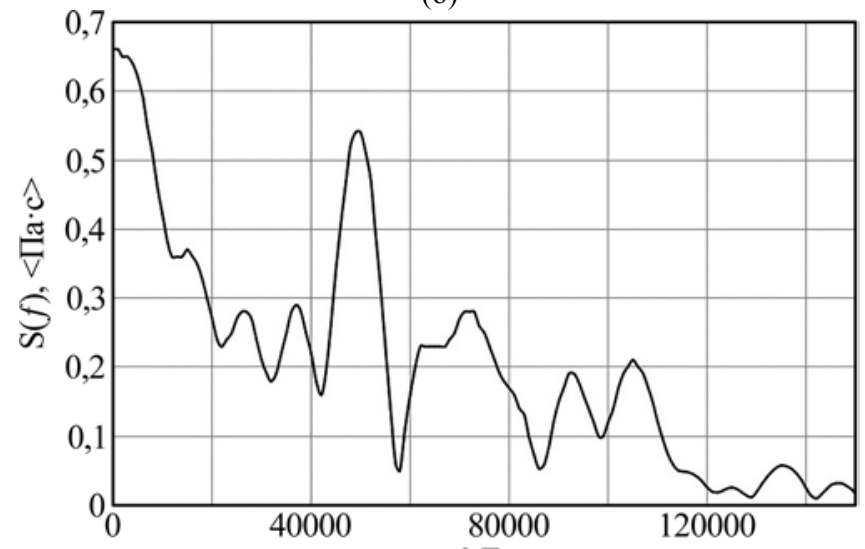

(в)

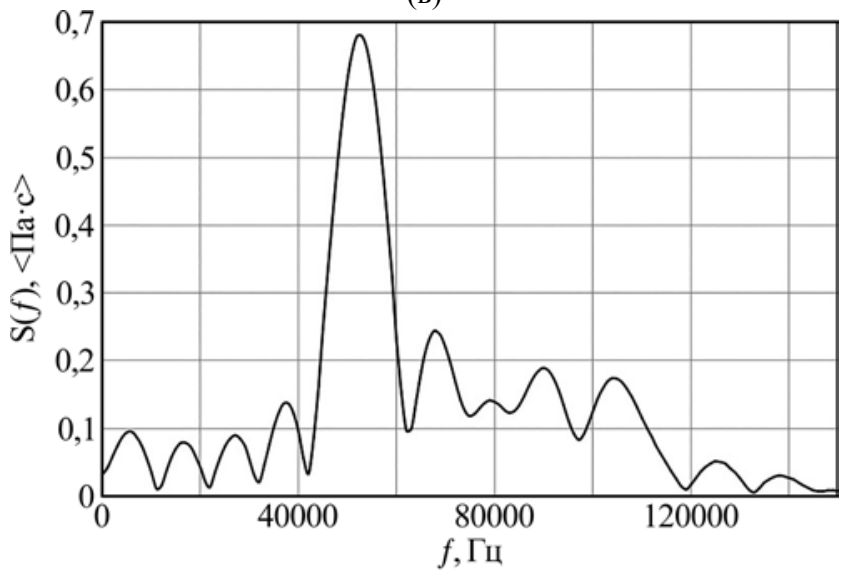

(г)

Рис. 5. Зависимости спектральной плотности давления в точках $A$ и $B$, размеры емкости с расплавом $h=0,09$ м, $r=0,04$ м. (a) - спектральная плотность в точке $A$ (период тока 2,8 мкс); (б) - спектральная плотность в точке $B$ (период тока 2,8 мкс); (в) - спектральная плотность в точке $A$ (период тока 28 мкс); (г) - спектральная плотность в точке $B$ (период тока 28 мкс). 


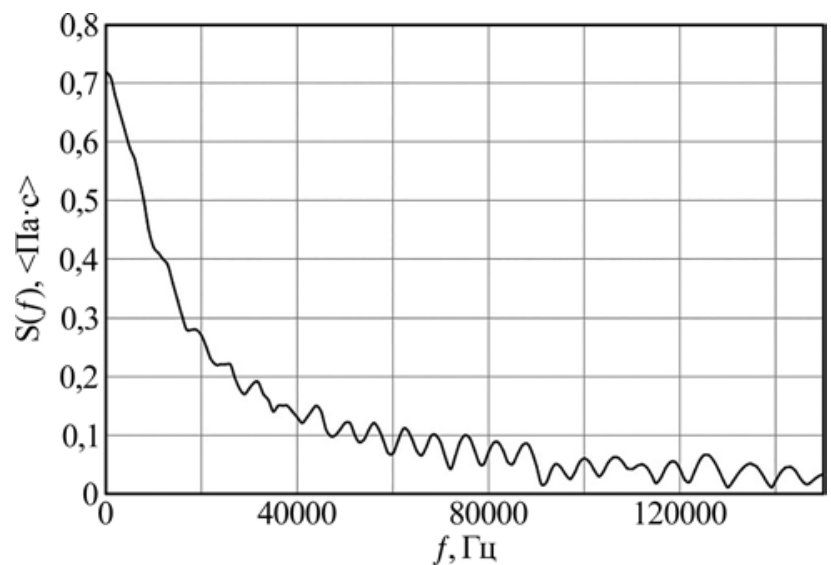

(a)

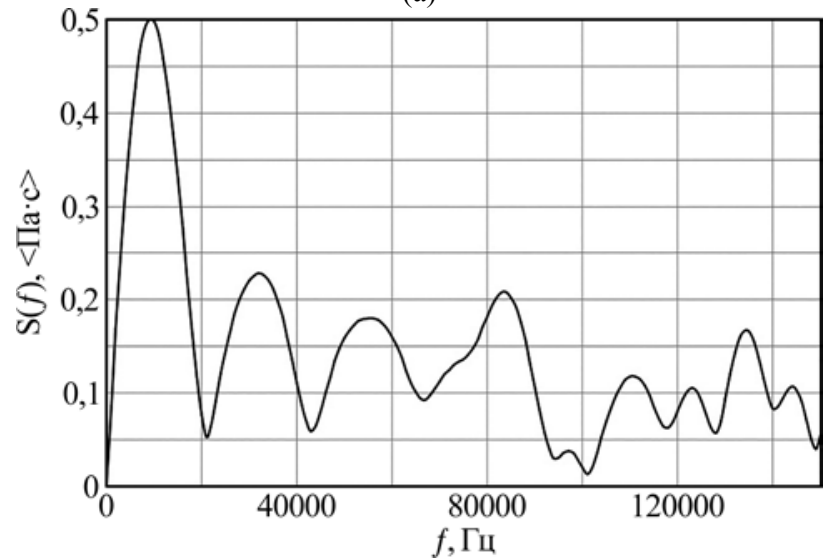

(б)

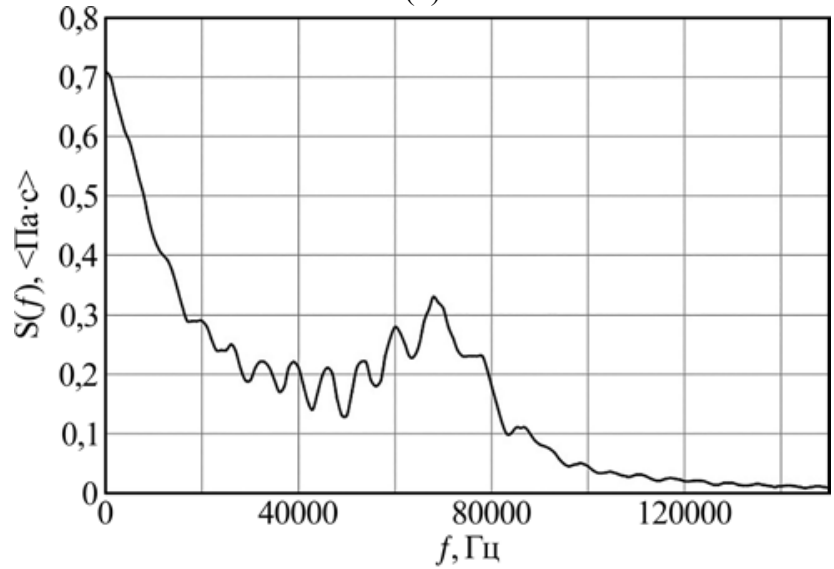

(в)

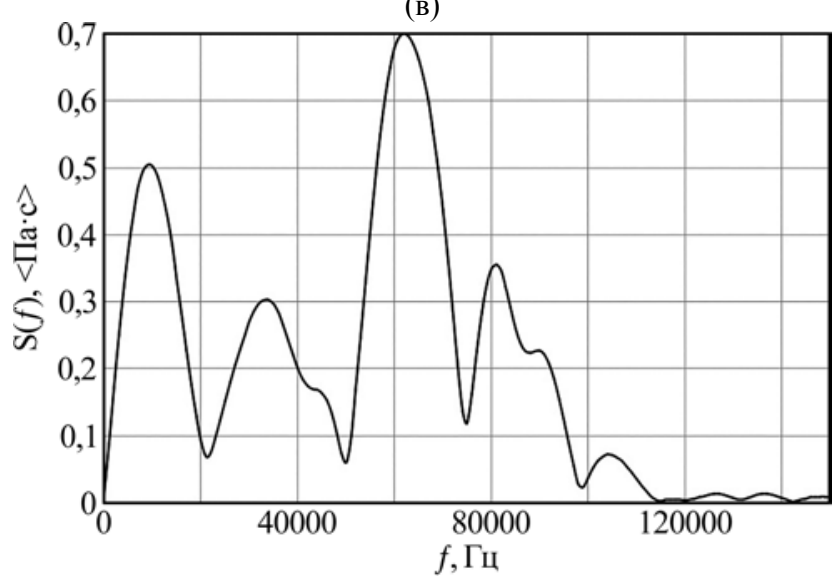

(г)

Рис. 6. Зависимости спектральной плотности давления в точках $A$ и $B$, период тока 28 мкс, размеры емкости с расплавом $h=0,9$ м, $r=0,4$ м. (а) - спектральная плотность в точке $A$ (период тока 2,8 мкс); (б) - спектральная плотность в точке $B$ (период тока 2,8 мкс); (в) - спектральная плотность в точке $A$ (период тока 28 мкс); (г) - спектральная плотность в точке $B$ (период тока 28 мкс). 
звуковой энергии растет. Жидкость сжимается, генерируя в этом объеме синхронные колебания давления [9].

На рис. 3 и 4 представлены временные зависимости первичного сигнального давления в точке $A$ и давления в точке $B$ для анализируемых вариантов. На рис. 5 и 6 - соответствующие спектры сигналов.

Как показано на рис. 3 и 4, амплитуда первичного сигнального давления по мере его распространения от стенки емкости (точка $A$ ) к центру (точка $B$ ) возрастает за счет увеличения плотности звуковой энергии, что характерно для цилиндра. Естественно, при этом конечные характеристики давления в районе оси емкости (точка $B$ ) существенно зависят от периода тока и размеров емкости. Такой эффект, например, активно используется в организации плазменных процессов [15]. Как отмечалось в работах [5-7, 10, 11], действие ЭМАП следует активно анализировать с точки зрения резонансов на модах собственных колебательных явлений флуктуирующих элементов жидкости (кластеры, первые координационные сферы, микро- или макровключения). Авторскими коллективами этих работ выполнены расчеты собственных колебаний для первой координационной сферы и кластера в различных вариантах колебаний при температуре кристаллизации. Они лежат в пределах:

- кооперативные вращательные - от 10 до $10^{3}$ кГц;

- вращательно-поступательные - от 1 до $10^{4}$ кГц;

- вращательные первой координационной сферы - от 1 до $10^{5}$ кГц.

В этом плане логично проанализировать спектры сигналов, представленных на рис. 5 и 6. При этом важно отметить, что импульс давления, в отличие от давления при ультразвуковой обработке, имеет широкий спектр амплитудночастотной характеристики. То есть обладает большими функциональными возможностями организовывать резонансные эффекты [16].

Ранее в работе [17] были проанализированы условия формирования резонансных эффектов в металлических расплавах. При этом показано, что, несмотря на значительный прогресс, достигнутый в принципиальном понимании этого физического явления, его использование в практических целях литейного производства и при пояснении на его основе возможных эффектов, влияющих на перестройку структуры, еще ожидает своего решения.

\section{ОБСУЖДЕНИЕ РЕЗУЛЬТАТОВ}

Параметры ЭМАП энергии в системе «источник тока-скин-слой» оценены в результате приближений, но они физически оправданы. Полученные данные показали, что энергия, формирующая магнитное давление в скин-слое, может составлять долю $d$ порядка $10^{-4}-10^{-2}$ от величины энергии, запасенной в конденсаторной батарее. А ключевым параметром разрядной цепи, определяющим эту долю, является период разрядного тока, так что $d \sim \sqrt{T}$.

Магнитное давление (плотность энергии в скин-слое) $P_{M}(t)=I^{2}(t) / r^{2}$. Но в реальных условиях размер емкости $r$ может изменяться на один-два порядка, а величина силы тока $I$ - от сотен ампер до десятков килоампер, что обусловлено выбором параметров генератора. Поэтому можно рекомендовать при обработке расплава в различных вариантах соблюдать пропорциональные изменения величин $I$ и $r$.

Численный анализ акустических возмущений выявил существенную количественную и качественную разницу результатов в анализируемых вариантах.

Для малой массы расплава ( $\approx 1$ кг) в случае периода тока 2,8 мкс, а периода магнитного давления 1,4 мкс во всем объеме формируется сложная существенно нелинейная акустическая обстановка как в точке $A$, так и в точке $B$. Амплитуда давления в точке $B$ по сравнению с первичной волной увеличивается на порядок. Отрицательное давление может достигать нижнего порога кавитации.

Спектральный состав давлений в точках $A$ и $B$ заполнен на всем протяжении анализируемого участка (145 кГц). Характерные пики $\approx 58$ и $\approx 107$ кГц соответствуют периодичности распространения импульса между стенками емкости.

Для периода тока 28 мкс (давления 14 мкс) волновая обстановка существенно изменяется. Суперпозиция волн также имеет нелинейный (но менее выраженный) характер. В точке $B$ наблюдается увеличение амплитуды давления до 4 раз. Спектральный состав волн проявляется с большими значениями спектральной плотности.

Акустическое возмущение в емкости с массой расплава $\approx 100$ кг при обоих значениях периода тока и качественно, и количественно существенно отличается от ситуации в емкости $\approx 1$ кг. Здесь для периода тока 2,8 мкс в точке $B$ период колебаний волны давления, очевидно, формируется не только суперпозицией волн, приходящих от скин-слоя, но и процессами, которые возникают перед их фронтами за счет эффекта сжимаемости жидкости. Степень нелинейности возмущений в центре емкости существенно уступает по значимости области скинслоя. 
Спектральный состав акустических возмущений разнится от аналогичной ситуации в малой емкости и имеет явный энергетический всплеск на частоте $\approx 9$ кГц, что соответствует супер-позиции волн на оси емкости.

Для периода тока 28 мкс качественная картина акустических возмущений в районе оси емкости отличается от 2,8 мкс несущественно. Чего нельзя сказать о ситуации в скин-слое. Хотя спектральный состав волн показывает явные количественные отличия за счет максимума в районе $\approx 62$ кГц, что соответствует периоду давления 14 мкс. Кроме того, практически после 100 кГц энергетическая эффективность мод незначительна.

Таким образом, волновая обстановка в расплаве при ЭМАП также существенно зависит от периода разрядного тока (циклической частоты) и размеров емкости с расплавом.

Учитывая мультифизические признаки ЭМАП, изучение его фундаментальных принципов еще далеко до завершения. Но решение отдельных задач позволяет определить некоторые особенности этого явления в прикладных аспектах. Полученные в данной статье результаты также позволяют предлагать некоторые общие рекомендации применительно к технологиям литейного производства в условиях ЭМАП в электротоковом режиме.

При постановке задачи ключевым параметром является геометрия емкости, при решении задачи - параметр скин-слоя и временная зависимость разрядного тока, что определяет выбор необходимого источника тока.

Важно отметить, что функциональные возможности ЭМАП зависят от совместного влияния на расплав последовательно электромагнитного и акустического воздействий. Этот вопрос требует отдельного всестороннего анализа.

\section{ВЫВОДЫ}

В работе на основе физических обоснований сделаны количественные оценки характеристик ЭМАП энергии в сквозной задаче для симметричного случая. Показано ключевое значение периода тока, продолжительности его импульса и размеров емкости с расплавом для эффектов ЭМАП, которые формируют его функциональные возможности.

В отличие от сферы влияния на расплав электромагнитного поля, ограниченной скинслоем, акустическое поле при определенных условиях охватывает весь объем расплава. Суммарный эффект воздействия для управления структурообразованием можно оценить по результатам работы вплоть до определения суммарного экстремума.

Авторы статьи надеются, что полученные данные помогут не только глубже понять функциональные возможности ЭМАП, но и найти оптимальные алгоритмы обработки расплава импульсами электрического тока в рамках концепции управления этими процессами.

\section{КОНФЛИКТ ИНТЕРЕСОВ}

Авторы заявляют, что у них нет конфликта интересов.

\section{ЛИТЕРАТУРА}

1. Pericleous, K., Bojarevics, V., Djambazov, G., Dybalska, A., et al., Contactless ultrasonic cavitation in alloy melts, Materials, 2019, vol. 12, p. 3610. doi:10.3390/ma12213610.

2. Timoshkin, I.Yu., Nikitin, K.V., Nikitin, V.I., Deev, V.B., Influence of melt processing with electromagnetic acoustic fields on the structure and properties of Al-Si system alloys, Proceedings Non-Ferrous Metallurgy, 2016, vol. 3, p. 28.

3. Глущенков, В.А., Никитин, В.И., Черников, Д.Г., Никитин, К.В., О воздействии импульсных магнитных полей на расплавы, Металлургия машиностроения, 2012, № 4, с. 47.

4. Иванов, А.В., Череповский, С.С., Особенности преобразования энергии в системе «Индукторрасплав» при магнитно-импульсной обработке расплавов, Вестник НТУ «ХПИ», 2015, № 48, с. 13.

5. Зарембо, В.И., Киселёва, О.Л., Колесников, А.А., Подгородская, Е.С., и др., Влияние импульсов тока на процессы плавления и кристаллизации металлов, Металлургия машиностроения, 2005, № 1 , c. 11.

6. Зарембо, В.И., Подгородская, Е.С., Колесников, А.А., Изменение ликвации жаропрочных сплавов на основе никеля и кобальта при кристаллизации в слабых электромагнитных полях в токовом режиме, Химическая промышленность, 2003, т. 80, № 9, с. 31.

7. Киселёва, О.Л., Колесников, А.А., Зарембо, В.И., Увеличение скоростей гетерогенных физикохимических превращений в режиме резонансного электромагнитно-акустического преобразования, Химическая промышиленность, 2003, т. 80, № 5, c. 12.

8. Бибиков, А.М., Халтурин, И.П., Зарембо, В.И., Управление структурообразованием и свойствами литых материалов слабым акустическим воздействием, Литейное производство, 2007, № 5, c. 12.

9. Коровин, В.И., Импульс давления в жидком проводнике цилиндрической формы, вызываемый импульсом тока, Журнал технической физики, 2005 , т. 75 , № 7 , c. 1 . 
10. Каганов, М.И., Васильев, А.Н., Электромагнитноакустическое преобразование - результат действия поверхностной силы, Успехи физических наук, 1993, т. 163, № 10, с. 67.

11. Васильев, А.Н., Каганов, М.И., Маллави, Ф.Н., Термоупругие напряжения - один из механизмов электромагнитно-акустического преобразования, Успехи физических наук, 1993, т. 163, № 10, с. 81.

12. Ivanov, A.V., Tsurkin, V.N., Peculiarities of distribution of electromagnetic current treatment of melts in different modes, Surf. Eng. Appl. Electrochem, 2018, vol. 55, no. 1, p. 53.

13. Гитис, М.Б., Михайлов, И.Г., Распространение звука в жидких металлах. Обзор, Акустический журнал, 1966, т. 12, № 2, с. 145.

14. Бойко, Н.И., Евдошенко, Л.С., Зароченцев, А.И., Иванов, В.М., Высоковольтные установки и технологии на основе комплекса высоковольтных импульсных воздействий, Вестник НТУ «ХПИ», 2004, № 35, c. 54.

15. Кадомцев, Б.Б., Коллективнье явления в плазме. М.: Наука, 1975. 303 с.

16. Харкевич, А.А., Спектры и анализ. М.: Физматгиз, $1962.236 \mathrm{c}$.

17. Цуркин, В.Н., К вопросу о резонансных процессах при обработке расплава в технологиях литейного производства, Проиессы литья, 2012, № 6, с. 30.

\section{Summary}

The paper deals with a symmetric problem on the base of physically substantiated estimates of the processes of electromagnetic-acoustic transformations (EMAT) of energy during the flow of an electric current through a melt, the key parameters of the open problem of the system "Power source parameters - Parameters of the magnetic field and magnetic pressure of the skin layer Parameters of acoustic disturbances". It was shown that the key parameter when formulating the EMAT problem in technological applications is the geometry of the container with the object of processing and the material of the form. And when solving the problem, they are the parameter of the skin layer and the time dependence of the discharge current. It was established that a part of energy during the formation of the magnetic pressure in the skin layer from the amount of the energy stored in the capacitor bank of the pulse current generator is on the order of $10^{-4}-10^{-2}$. The value of this part depends on the period of the discharge current and is proportional to the $\sqrt{\mathrm{T}}$. When acoustic disturbances propagate in a melt, the main share of energy losses is determined by the difference in the acoustic stiffness of the melt and the shape of materials. The frequency spectra of the pressure of sound waves at the parameters selected for the analysis can cover the range of up to hundreds $\mathrm{kHz}$, which is a good reason for the realization of resonance effects and the active formation of dissipative structures. Attention is focused on the fact that EMAT effects are manifested in the melt not only under the influence of an acoustic field, but also under that of an electromagnetic one in the skin layer. They are separated in time, but the acoustic field can occupy the entire volume of the melt and its effect is longer in time.

Keywords: electric discharge, melt, energy conversion, energy losses, acoustic wave processes, functional capabilities 